\title{
Elicitation of expert prior opinion: application to the mypan trial in childhood polyarteritis nodosa
}

\author{
Lisa Hampson', John Whitehead', Despina Eleftheriou², Catherine Tudur-Smith ${ }^{3}$, Rachel Jones ${ }^{4}$, David Jayne ${ }^{4}$, \\ Helen Hickey ${ }^{5}$, Paul Brogan ${ }^{2 *}$, MYPAN vasculitis expert group
}

From 21st European Pediatric Rheumatology (PReS) Congress

Belgrade, Serbia. 17-21 September 2014

\section{Introduction}

A major challenge in rare diseases is conducting clinical trials with sufficient power to inform best clinical practice when anticipated sample sizes are small. Historically, this has been a major barrier in rare paediatric autoimmune diseases. Bayesian methodology can be used to augment the sparse therapeutic data obtained from clinical trials in these circumstances.

\section{Objectives}

We elicited expert prior opinion for a future Bayesian randomised controlled trial for a rare inflammatory paediatric disease, polyarteritis nodosa (MYPAN, Mycophenolate mofetil for polyarteritis nodosa).

\section{Methods}

A Bayesian prior elicitation meeting was convened. Participating experts were drawn from across the EU and Turkey. Opinion was sought on the probability that a patient in the MYPAN trial treated with cyclophosphamide would achieve disease remission within 6-months, and on the relative efficacies of mycophenolate mofetil and cyclophosphamide. Expert opinion was combined with previously unseen data from a recently completed randomised controlled trial of mycophenolate mofetil versus cyclophosphamide in anti-neutrophil cytoplasmic antibody associated vasculitis.

\section{Results}

A pan-European group of fifteen experts participated in the elicitation meeting. Consensus expert prior opinion was that the most likely rates of disease remission within 6 months on cyclophosphamide or mycophenolate mofetil were $74 \%$ and $71 \%$ respectively. This prior opinion will now be taken in to account and will be modified to formulate a Bayesian posterior opinion when data from 40 patients completing the trial randomised at a 1:1 ratio to either receive cyclophosphamide or mycophenolate mofetil are available.

\section{Conclusion}

We suggest that this methodological template could be applied to trial design for other rare diseases, and is of particular relevance to rare autoimmune conditions that currently lack a good evidence base for treatment.

\section{Disclosure of interest}

None declared.

\section{Authors' details}

'Department of Mathematics and Statistics, Lancaster University, Lancaster, UK. ${ }^{2}$ Paediatric Rheumatology, Institute of Child Health, London, UK. ${ }^{3} \mathrm{MRC}$ North West Hub for Trials Methodology Research, Department of Biostatistics, Liverpool University, Liverpool, UK. ${ }^{4}$ Department of Renal Medicine, Addenbrookes Hospital, Cambridge, UK. ${ }^{5}$ Medicines for Children Research Network Clinical Trials Unit, University of Liverpool, Liverpool, UK.

Published: 17 September 2014

doi:10.1186/1546-0096-12-S1-P125

Cite this article as: Hampson et al.: Elicitation of expert prior opinion: application to the mypan trial in childhood polyarteritis nodosa. Pediatric Rheumatology 2014 12(Suppl 1):P125.

${ }^{2}$ Paediatric Rheumatology, Institute of Child Health, London, UK

Full list of author information is available at the end of the article 